\title{
MEJORANDO LAS COMPETENCIAS PARA LA ENSEÑANZA DE LA ESTADÍSTICA DE PROFESORES DE SECUNDARIA EN FORMACIÓN A TRAVÉS DE TALLERES PARTICIPATIVOS
}

\author{
$\underline{\text { Adriana Pérez }}{ }^{1}$, Gerardo Cueto ${ }^{1}$, María Soledad Fernández ${ }^{1}$, Julieta Filloy ${ }^{1}$, Stella Maris Diez ${ }^{2}$, \\ Diana Kelmansky ${ }^{1}$, Carlos Pomilio ${ }^{1}$ \\ ${ }^{1}$ Universidad de Buenos Aires (UBA), Argentina \\ ${ }^{2}$ Universidad de Palermo, Argentina \\ aaperez@ege.fcen.uba.ar
}

Se describe una intervención didáctica cuyo objetivo fue acompañar el trayecto formativo en estadística de los futuros profesores de Matemática de la escuela media. Se diseñaron talleres sobre estadistica descriptiva y probabilidad que comprendieron una revisión conceptual, la identificación de los obstáculos epistemológicos, el desarrollo de actividades didácticas y un espacio de reflexión final. Las actividades incluyeron material manipulativo, simulaciones y análisis de datos reales. Se dictaron 5 talleres en institutos de formación docente de Buenos Aires. Los 280 asistentes mostraron un alto grado de participación y evaluaron positivamente la iniciativa en cuanto a contenidos, pertinencia y aplicabilidad. Se diseñó una página web para facilitar la comunicación e interacción con la comunidad educativa. Esta iniciativa promueve el razonamiento estadístico y una mejor actitud hacia la estadística en los profesores, lo cual es imprescindible si se desea obtener egresados de la escuela media capaces de interpretar información estadística de manera fundamentada, critica e independiente.

\section{DESCRIPCION DEL PROBLEMA}

En los últimos años se han registrado numerosos esfuerzos tendientes a promover la enseñanza de la estadística en el nivel medio, tales como cambios en los contenidos e incorporación de TIC. En Argentina, en etapas sucesivas entre 2004 y 2011, se elaboraron y aprobaron por el Consejo Federal de Educación los Núcleos de Aprendizajes Prioritarios (NAP) para la educación secundaria en Argentina. Los NAP plasman los saberes considerados claves, relevantes y significativos para la sociedad, en tanto preparan a nuestros jóvenes para una vida y participación democrática. En particular, resultan relevantes para el caso que nos ocupa los NAP del área de matemática de educación secundaria, que incluyen formalmente probabilidad y estadística como contenidos prioritarios.

No obstante la trascendencia de los acuerdos logrados, la realidad nos muestra que actualmente en Argentina la formación en estadística de los egresados del nivel medio adolece de serias deficiencias que se manifiestan en el uso posterior de la estadística en su vida cotidiana así como en los ámbitos universitario y profesional, y según entendemos este fenómeno, en buena parte, puede explicarse por la propia formación y desempeño de otro actor clave, el profesor de matemática.

En efecto, la formación en estadística de los futuros profesores de matemática presenta en líneas generales varias limitaciones. Los planes de estudio de los institutos de formación docente incluyen una única materia -Probabilidad y Estadística- cuyos contenidos se limitan a estadística descriptiva y nociones básicas de probabilidad. El enfoque de su enseñanza es en general abstracto, vinculado a los aspectos matemáticos y procedimentales. Este enfoque, además de ser menos efectivo para el aprendizaje de la estadística que el de entornos orientados a la solución de problemas basados en datos (Gal, 2002), lleva a que muchas veces las creencias arraigadas sobre la naturaleza determinista y jerárquicamente estructurada de la matemática sean transferidas a la enseñanza de la estadística, ignorando su naturaleza estocástica (Meletiou, 2007). Además, no se imparte un entrenamiento formal en un software estadístico desaprovechando las potencialidades que ofrecen estos medios tecnológicos como recurso didáctico o herramienta de trabajo, ni se ofrece formación específica en didáctica de la estadística.

Para el docente en actividad, las posibilidades de capacitación también son escasas. De la oferta brindada por la Escuela de Capacitación docente dependiente del Ministerio de Educación del Gobierno de la Ciudad de Buenos Aires y uno de los principales centros de formación docente continua de Latinoamérica, solo un curso de los más de 50 dirigidos al nivel medio, aborda

In: M.A. Sorto (Ed.), Advances in statistics education: developments, experiences and assessments. Proceedings of the Satellite conference of the International Association for Statistical Education (IASE), July 2015, Rio de Janeiro, Brazil. (C)2015 ISI/IASE iase-web.org/Conference_Proceedings.php 
contenidos de estadística. Otro antecedente es un curso de formación a distancia denominado Alfabetización estadística en la escuela obligatoria argentina, ofrecido en dos oportunidades por la Universidad Nacional del Litoral.

Por otra parte, los libros de texto, en tanto recursos didácticos como fuente de conocimientos para estudiantes y docentes, también cumplen un rol central en la formación y práctica docente. Es frecuente que los profesores los perciban como un mandato, prescribiendo y dirigiendo la enseñanza (Reys, Reys y Chavez, 2004). Siguiendo la taxonomía de Bloom (Anderson y Krathwohl, 2001) para la clasificación de las metas educacionales se catalogaron las actividades relacionadas con estadística descriptiva y probabilidades de los diez libros de texto de matemática más utilizados en el nivel medio. Se observó que las destrezas cognitivas activadas pertenecían en su mayoría a habilidades de pensamiento de orden inferior (recordar, comprender) o medio (aplicar) y solo un 15\% de la ejercitación proponía actividades de orden superior (analizar, evaluar, crear) (datos no publicados). En resumen, los libros de texto disponibles ofrecen mayormente una visión mecanicista de la estadística, con ejercitación descontextualizada y reiterativa, orientada a lo procedimental y sin objetivos específicos, por lo se deduce que tampoco contribuyen a una mejor formación docente.

No sorprende entonces que una prueba sobre conocimientos estadísticos efectuada en estudiantes de último año de 10 de los 26 institutos de formación de profesores de matemática de Buenos Aires arrojó solo un $42 \%$ de respuestas correctas (Fabrizio y cols, 2007). Los futuros profesores evidenciaron serias dificultades en la interpretación de gráficos, estadísticos de tendencia central y de variabilidad, así como de probabilidades. Por otro lado, sólo un $26 \%$ declaró haber utilizado una computadora durante el curso de Probabilidad y Estadística. Tauber et al. (2013) también hallaron dificultades en la resolución de actividades que implicasen razonamiento estadístico por parte de profesores de matemática en formación y en ejercicio.

Esta escasa preparación en la disciplina con la que el profesor termina sus estudios hace que cuente con pocos recursos para su enseñanza y evidencia una actitud negativa hacia la disciplina. En ese sentido, Estrada (2004) halló que la actitud hacia la estadística del profesor en ejercicio se deteriora con la práctica docente, debido a la dificultad que él mismo encuentra en la disciplina, a la escasa importancia que se le otorga o a la dificultad para aprender que aprecia en sus alumnos. Como consecuencia, los contenidos de estadística no son habitualmente desarrollados en el ciclo lectivo o bien quedan reducidos a unas pocas clases, con un tratamiento habitualmente limitado a los aspectos procedimentales. No sorprende entonces que una encuesta efectuada a 50 profesores de matemática de escuelas públicas y privadas de Buenos Aires arrojó que, si bien el $80 \%$ incluyó nociones de estadística en la planificación anual, el 70\% de los que la incluyeron no alcanzaron a desarrollar los temas en el curso lectivo. Adicionalmente, el 66\% manifestó que no se consideraba capacitado para desarrollar exitosamente los temas de estadística incluidos en los contenidos curriculares (Kucukbeyaz y Batto, 2012). Similares observaciones han sido documentadas en otras universidades nacionales (Fernández de Carrera, 2002).

Como consecuencia de lo anteriormente expuesto, muchos profesores de matemática egresan con una visión sesgada de la estadística, con un dominio limitado de las herramientas estadísticas, escasos recursos didácticos para la enseñanza de la disciplina, una actitud negativa y limitadas oportunidades de formación profesional, con lo que se ponen en riesgo los propios objetivos del sistema educativo.

La problemática descripta no es exclusiva de nuestro país (Meletiou, 2007, Batanero, 2009). Existe un creciente interés a nivel internacional sobre la formación en estadística de los profesores y su impacto sobre la calidad del proceso de enseñanza-aprendizaje de la disciplina (veáse Batanero, Burrill y Reading, 2011). Queremos creer que el escenario actual es de transición. También creemos en el valor de sostener el proceso a través de contribuciones de otros actores comprometidos.

En este contexto, se llevó a cabo una intervención didáctica con los siguientes objetivos:

- acompañar el trayecto formativo en estadística de futuros profesores de matemática, mejorando sus habilidades y actitudes hacia la disciplina, y

- desarrollar y difundir recursos y materiales didácticos pertinentes.

La presente comunicación da cuenta de la experiencia y de los resultados preliminares de la intervención realizada. 


\section{DISEÑO E IMPLEMENTACION DE TALLERES}

La intervención estuvo dirigida a estudiantes que se encontraban cursando el 3er o 4to año de la carrera de profesor de matemática de educación secundaria en institutos de formación docente públicos, y a sus docentes. El dictado de los talleres se articuló con la planificación de Probabilidad y Estadística, de manera que los estudiantes ya habían recibido la formación teórica en los temas abordados en los talleres.

El equipo de trabajo estuvo constituido por docentes con larga experiencia en la enseñanza de la estadística en carreras no matemáticas del nivel universitario.

Se diseñaron dos talleres, que abordaron los tópicos estadísticos que integran los contenidos curriculares del nivel medio (Ministerio de Educación, 2011):

- Taller 1: "Muestras, gráficos y estadísticos en la descripción de un fenómeno de interés", que incluyó recopilación de datos, representaciones tabulares y gráficas y estadísticos descriptivos

- Taller 2: "Hagan sus apuestas. El azar y la probabilidad en la vida cotidiana", sobre fenómenos aleatorios y cálculo de probabilidades.

La estructura de los talleres fue la siguiente:

- Introducción: Los talleres comenzaron con una introducción realizada por el formador de profesores, quien describe los objetivos del taller y efectúa una breve revisión conceptual.

- Identificación de los obstáculos epistemológicos, preconceptos e ideas presentes en los estudiantes: Se discutieron las principales dificultades en el proceso de enseñanza-aprendizaje documentadas por la bibliografía (ver por ejemplo Batanero, 2001).

- Desarrollo de actividades didácticas: Se propuso a los futuros profesores experimentar una situación didáctica en la que cumplirían el rol de alumnos y el formador de profesores el rol del profesor. Para la selección de las actividades se tuvieron en cuenta las recomendaciones de la American Statistical Association para la enseñanza de estadística en el nivel medio (Guidelines for Assessment and Instruction in Statistics Education (GAISE) College Report) (Garfield et al., 2005) y las de Burrill y Biehler (2011). Las actividades, de modalidad participativa, incluyeron juegos, simulaciones, análisis con datos reales, discusión de casos y uso de TIC y estuvieron orientadas a promover la alfabetización y el razonamiento estadísticos.

A modo de ejemplo, en el taller de Probabilidades, se trabajó con el problema de Monty Hall, juegos de lotería y rachas en el lanzamiento de monedas. En el taller de descriptiva se trabajó con datos reales generados en el aula y lectura crítica de información publicada en medios, entre otros. Una descripción más exhaustiva de los talleres y del material didáctico desarrollado se encuentra disponible en:

https://alfabetizacionestadistica.wordpress.com/nuestros-talleres/

\section{IMPLEMENTACIÓN Y RESULTADOS}

Se dictaron 5 talleres en institutos de formación docente de Buenos Aires, con 280 asistentes. Cada taller tuvo una duración de $3 \mathrm{hs}$. Al finalizar cada taller se estableció un espacio de reflexión con los asistentes, indagando sus pareceres sobre las actividades propuestas, su aplicabilidad y relevancia y sobre la importancia de la formación en estadística. Dado que gran parte de los asistentes contaban con experiencia de trabajo en el aula como parte de las prácticas profesionales, este intercambio de saberes entre actores del nivel medio y universitario permitió un enriquecimiento y reelaboración de las propuestas iniciales. Los cambios sugeridos apuntaron fundamentalmente a darle mayor dinamismo a los talleres, con menor desarrollo expositivo y más foco en las actividades propiamente dichas.

La evaluación de la intervención fue altamente favorable. Los estudiantes mostraron un muy alto grado de participación en las actividades y en los espacios de discusión. Si bien en el desarrollo de los talleres se expusieron las principales dificultades en el apropiamiento de los conceptos estadísticos, las actividades didácticas permitieron poner en evidencia ideas erróneas por parte de los futuros profesores.

Al finalizar los talleres se efectuaron encuestas anónimas y voluntarias para indagar la opinión de los asistentes acerca de la utilidad de los talleres en relación al aporte de conocimientos y la factibilidad de aplicarlos en el aula con los estudiantes de nivel medio. Las primeras cinco preguntas efectuadas se respondían mediante una escala Likert, con una valoración de 1 
(totalmente en desacuerdo) a 5 (totalmente de acuerdo). La última pregunta consistió en realizar una valoración general del taller y debía ser respondida utilizando una escala de puntuación de 1 (peor nota) a 10 (mejor nota). Los resultados se muestran en la Tabla 1.

Tabla 1. Resultados porcentuales de la encuesta efectuada a los asistentes a los talleres Respuesta

\begin{tabular}{ccccc}
\hline 1 & 2 & 3 & 4 & 5 \\
$\begin{array}{c}\text { Totalmente } \\
\text { en }\end{array}$ & $\begin{array}{c}\text { Parcialmente } \\
\text { en }\end{array}$ & $\begin{array}{c}\text { Ni de } \\
\text { acuerdo ni } \\
\text { en }\end{array}$ & $\begin{array}{c}\text { Parcialmente } \\
\text { de acuerdo }\end{array}$ & $\begin{array}{c}\text { Totalmente } \\
\text { de acuerdo }\end{array}$ \\
desacuerdo & desacuerdo & $\begin{array}{c}\text { esacuerdo } \\
\text { desalo }\end{array}$ & & \\
\hline
\end{tabular}

P1. El taller contribuyó a mejorar mi entendimiento $1,4 \%$ $2,1 \%$ $8,2 \%$ $25,3 \%$ $63,0 \%$ sobre los temas estadísticos P2. Las actividades que se desarrollaron en el taller son pertinentes para la enseñanza $1,4 \%$ $2,0 \%$ $16,2 \%$ $35,1 \%$ $45,3 \%$ de estadística en la escuela P3. Las actividades que se desarrollaron en el taller son factibles de aplicar en la escuela

\begin{tabular}{lccccc}
\hline P4. Las aplicaría & $1,4 \%$ & $3,4 \%$ & $22,3 \%$ & $33,8 \%$ & $39,2 \%$ \\
\hline $\begin{array}{l}\text { P5. El taller contribuyó a } \\
\text { mejorar mi actitud hacia la } \\
\text { estadística. }\end{array}$ & $1,4 \%$ & $2,0 \%$ & $8,8 \%$ & $33,1 \%$ & $54,7 \%$ \\
\hline
\end{tabular}

Se observó que casi el 90\% de los asistentes consideró que el taller contribuyó a mejorar el entendimiento de los tópicos estadísticos abordados (P1) y su actitud hacia la estadística (P5). Un $80 \%$ consideró que las actividades desarrolladas son pertinentes para la enseñanza de estadística en la escuela (P2), pero estos valores descienden al 71 y $73 \%$ respectivamente cuando se indaga sobre su factibilidad, en general (P3) y en la propia práctica docente (P4). Si bien estos valores son elevados, evidencian las dificultades percibidas por los futuros profesores para conectar sus conocimientos con la práctica docente.

Finalmente, frente a la pregunta "¿Qué valoración final harías de las actividades del taller (de 1 a 10)?", se obtuvo una mediana de 9 con un rango de 5 a 10 y un promedio de 8,66 con un coeficiente de variación del $13 \%$.

En cuanto a los formadores de los futuros profesores, responsables del dictado de Probabilidad y Estadística, cumplieron un rol fundamental como aliados en el desarrollo del proyecto. Actuaron como nexo entre la comunidad del nivel medio y el equipo universitario. Valoraron especialmente el enfoque ("la metodología que ustedes proponen permite ver claramente la importancia de tener conocimientos de la estadística descriptiva para el análisis crítico de cierta información") y el nexo con la universidad ("Lo interesante del proyecto es el intercambio y acercamiento que se produce para nuestros alumnos con docentes del nivel universitario que están especializados en la aplicación de un área como las Probabilidades y la estadística permitiendo a los docentes en formación tener un intercambio concreto con dichos especialistas").

En resumen, estos resultados indican una valoración altamente positiva de la propuesta pedagógica, en cuanto a contenidos, pertinencia y, en menor medida, en cuanto a su aplicabilidad.

Finalmente, se implementó una página WEB (http://alfabetizacionestadistica.wordpress.com) con el objetivo de difundir la propuesta y acercar a los interesados el material didáctico. Asimismo se generó una base de datos con los correos electrónicos de los asistentes a nuestros talleres. 


\section{CONCLUSIONES}

Los profesores cumplen un rol esencial en la adaptación y ejecución de los contenidos en el aula. La evidencia disponible indica fuertemente que el cambio en la enseñanza de la estadística en la escuela media dependerá en gran medida de un cambio en la formación y en la actitud de los profesores de matemática, quienes explícitamente abordan los contenidos estadísticos, y en ese sentido elaboramos el presente proyecto.

Creemos que es necesario impulsar cambios en tres dimensiones en el curriculum de la carrera de profesor de matemática: más contenidos estadísticos, un enfoque de enseñanza orientado al razonamiento estadístico más que a los aspectos procedimentales y la incorporación de la didáctica de la estadística en el campo de formación específico.

En esta línea es que implementamos una intervención orientada al universo de los profesores de matemática del nivel medio, y en particular a los profesores en formación, bajo el supuesto que el éxito de la intervención sería mayor por cuanto los estudiantes son de más fácil acceso, la posibilidad de lograr cambios actitudinales es mayor y el efecto multiplicador será mayor.

Los resultados preliminares sugieren que la intervención favorece una actitud positiva hacia la estadística y potencialmente a mejorar las competencias de los profesores de matemática en formación.

En la siguiente etapa del proyecto se planea su ampliación que incluye la implementación de un curso con mayor carga horaria, con más propuestas didácticas y espacios de reflexión sobre la propia experiencia matemática y sobre los procesos de enseñanza y aprendizaje. También se planea ampliar la red de institutos involucrados y potenciar la comunicación con la comunidad educativa a través de la creación de un grupo virtual mediado por una página web, que constituya un espacio de discusión e intercambio de información y recursos disponibles. Asimismo se prevé reunir evidencias sobre los cambios actitudinales y de habilidades y competencias, así como de su impacto en el mediano plazo.

\section{Agradecimientos}

Este proyectó contó con financiamiento del programa "Exactas con la Sociedad 4", dependiente de la Secretaría de Extensión y Bienestar Estudiantil de la Facultad de Ciencias Exactas y Naturales de la Universidad de Buenos Aires.

\section{REFERENCIAS}

Anderson, L. W. \& Krathwohl, D.R., et al (2001). A taxonomy for learning, teaching and assessing: A revision of Bloom's taxonomy of educational objectives. New York: Longman.

Batanero, C. (2001). Didáctica de la Estadística. Granada. Grupo de Investigación en Educación Estadística.

Batanero, C. (2009). Retos para la formación estadística de los profesores. II Encontro de Probabilidade e Estatística na Scola. Universidade do Minho, Portugal

Batanero, C., Burrill, G., \& Reading, C. (2011). Overview: challenges for teaching statistics in school mathematics and preparing mathematics teachers. Teaching Statistics in SchoolMathematics-Challenges for Teaching and Teacher Education: A Joint ICMI/IASE Study, 407-418.

Estrada, A., Batanero, C., \& Fortuny, J. M. (2004). Un estudio comparado de las actitudes hacia la estadística en profesores en formación y en ejercicio. Enseñanza de las Ciencias, 22(2), 263274.

Fabrizio, M.C., López, M.V., \& Plencovich, M.C. (2007) Statistics in Mathematics Teacher Training Colleges in Buenos Aires, Argentina: Assessment and Challenges. International Statistical Institute, 56th Session, Portugal

Fernández de Carrera, E. (2002) Teaching Statistics in secondary school. An overview: from the curriculum to reality. ICOTS 6 , Sudáfrica

Gal, I. (2002). Adults' statistical literacy: Meanings, components, responsibilities. International Statistical Review, 70(1), 1-25.

Garfield, J., Aliaga, M., Cobb, G., Cuff, C., Gould, R., Lock, R., et al. (2005). Guidelines for assessment and instruction in statistics education (GAISE) college report. Alexandria, VA: 
American Statistical Association. Online: www.amstat.org/education/gaise/

Kucukbeyaz D., \& Batto M. (2012). El desarrollo de métodos para la enseñanza de la Estadística en la educación media. X Congreso Latinoamericano de Sociedades de Estadística. Córdoba, Argentina.

Meletiou M. (2007). On the formalist view of mathematics: impact on. statistics instruction and learning. Mathematics Education Library, 42(6),131-155.

Ministerio de Educación, Consejo Federal de Educación (2011). Núcleos de Aprendizaje Prioritarios. Matemática. Buenos Aires.

Tauber, L., Cravero, M., \& Redondo, Y. (2013). Evaluación de errores de profesores de Matemática en tareas de Alfabetización Estadística y de Razonamiento Estadístico. En J. M. Contreras, G. R. Cañadas, M. M. Gea y P. Arteaga (Eds.), Actas de las Jornadas Virtuales en Didáctica de la Estadística, Probabilidad y Combinatoria (pp. 273-283). Granada, Departamento de Didáctica de la Matemática de la Universidad de Granada. 